\title{
3D Parametric Modeling and Finite Element Analysis of Yaw Bearing
}

\author{
Liu Yanping, Wang Yang \\ School of Energy \\ Power and Mechanical Engineering \\ North China Electric Power University \\ Beijing 102206
}

\author{
Zhou Fei \\ School of Energy \\ Power and Mechanical Engineering \\ North China Electric Power University \\ Beijing 102206
}

\begin{abstract}
This paper presented the process of using a function of the parametric design and assembly modes of UG software to realize the parametric design and modeling for the yaw bearing. The built 3-dimension model was imported into ANSYS. A new method was used to find the precisely loading position. Finally, the stress and strain of the gear teeth can be obtained through the finite element analysis. The results provided reference to optimize the design and improve the machining process of the yaw bearing.
\end{abstract}

Keywords- yaw bearing, 3D modeling, load scheme, finite element analysis

\section{INTRODUCTION}

With the worldwide lack of fossil energy, wind energy has developed rapidly as a kind of renewable clean energy. China has rich wind energy resource and strong financial support from the government, but the develpoment of wind power in China is reletively late, the relevant technology is behind the EU and US. For the yaw bearing, it is an important component of wind turbines. The domestic yawing bearings of MW wind tubines suffers high failure rate. So, its performance and useful life need to be studied and improved.

In this paper, the 3D model of the yaw bearing is accomplished by parametric solid modeling technology, and a new method of setting loads is applied in finite element analysis for more accurate load.

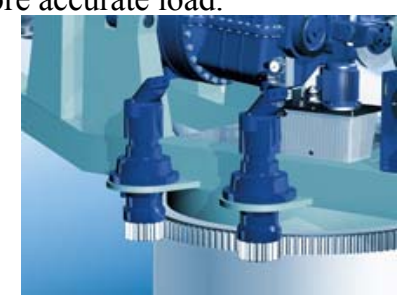

Figure 1. The yaw system

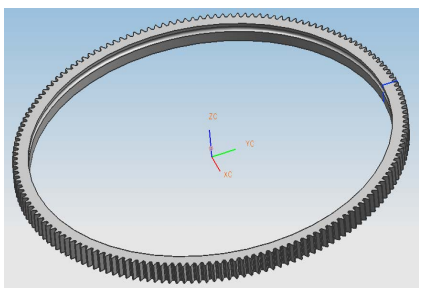

Figure 2. Model of the outer ring gear

\section{YAW SYSTERM}

In order to capture the wind power better and increase the effective working time, is equipped in modern wind turbines[1]. Generally, a yaw system consists of a yaw bearing, yaw actuator, yaw brake, counter and cable protection device, as shown in Figure 1.

\section{3-D PARAMETRIC MODELING}

$\mathrm{UG}$ is an advanced $\mathrm{CAD} / \mathrm{CAM} / \mathrm{CAE}$ software package developed by EDS Crop., which has a powerful modeling capability of designing complex entities [2]. So, UG is selected for 3D parametric modeling of the yaw bearing in our research. The specific modeling steps of the yaw bearing are listed as follows:

1) Use the rotating scanning function to set the inner ring and outer ring.

2) Use the parametric modeling capability to built the outer ring gear model[3],as shown in Fig. 2.

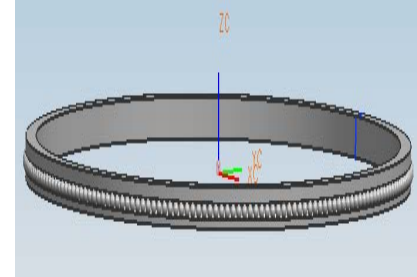

Figure 3. Model of the inner ring and the balls

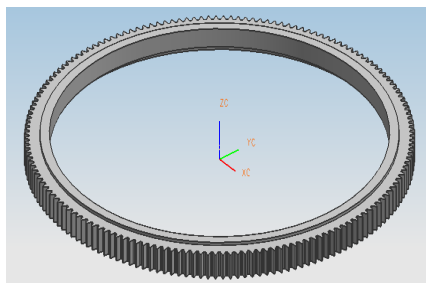

Figure 4. Model of the yaw bearing

3) Establish a rolling ball and pattern the ball around the $\mathrm{Z}$ axis to generate the other balls, as shown in Fig. 3 .

4) Assemble the components, as shown in Fig. 4. Since the yaw bearing is a four-point contact ball bearing and the clearance is tiny, the internal structure can easily be observed with the partial cutaway tool of UG, which may ensure the assembly accuracy. Then we can edit the design relationship 
and complete the parametric modeling. The assembly can adjust itself automatically when the parameters of the component change.

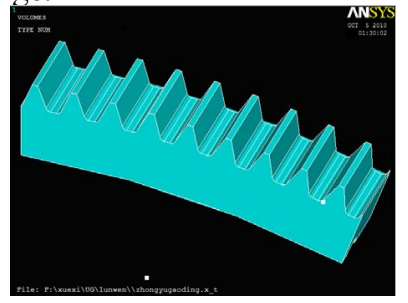

Figure 5. The model imported into ANSYS

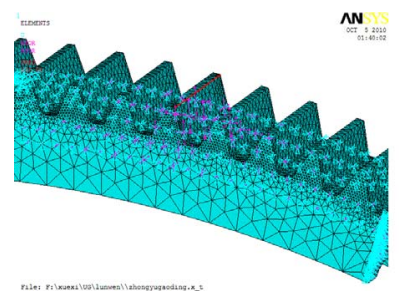

Figure 6. The model after loading and meshing

\section{FINITE ELEMENT ANALYSIS}

The yaw bearing should have high impact resistance, high reliability and useful life for 20 years[4]. Based on its working conditions, the ANSYS software is applied for finite element analysis on the yaw bearing to identify the weak part and provide a reference to optimize the design and improve the maching process [5]. According to the operating experience of wind farms, surface pitting, wearing and toothroot bending fatigue failure of the outer ring gear are the main failure forms. Thus, the the outer ring is chosen for the analysis.

1) Import the model into ANSYS

Because the bearing is symmetric, part of the gear is chosen to improve the efficiency of FEM calculating as shown in Fig. 5.

2) Set unit type and Meshing

In order to ensure the accuracy, the grids need to be accurate at the transition part from the tooth to tooth root circle as shown in Fig. 6. So, the structured solid with tet 10 node and 92 unit is selected here.

3) Set load

For FEA, the load setting is important. The traditional methods tend to choose "line-line" contact style. However, deformation occurres at the loading point during meshing of gears. So the contact area is actually a small plane. Besides, it is difficult for ANSYS to accurately determine the line load direction on tooth surface. So, the "face-face" contact style is chosen here and a new method is proposed to accurately determine the loading position. The specific steps are listed as follows:

(1) Determine the length of the involute

The length of the gear involute is the length of the line between the base circle and addendum circle. The involute equation in the Cartesian coordinate system can be written as:

$$
\left\{\begin{array}{l}
x=r_{b} \cos \varphi+r_{b} \varphi \sin \varphi \\
y=r_{b} \sin \varphi-r_{b} \varphi \cos \varphi
\end{array}\right.
$$

where $r_{b}$ is the radius of base circle and $\varphi$ is the parameter.

The integral of the curve length can be given by:

$$
L=\int_{\varphi 1}^{\varphi 2} \sqrt{x_{\varphi}^{\prime 2}+y_{\varphi}^{\prime 2}} d \varphi
$$

With

$$
\begin{aligned}
& x_{\varphi}^{\prime}=\frac{d x}{d \varphi}=r_{b} \varphi \cos \varphi \\
& y_{\varphi}^{\prime}=\frac{d y}{d \varphi}=r_{b} \varphi \sin \varphi
\end{aligned}
$$

where $L$ is the length of gear involute, $\varphi_{1}$ is the starting angle, and $\varphi_{2}$ is the termination angle.

Then, we can obtain:

$$
L=\int_{\varphi 1}^{\varphi 1} \sqrt{x_{\varphi}^{\prime 2}+y_{\varphi}^{\prime 2}} d \varphi=r_{b} \frac{\varphi_{1}^{2}+\varphi_{2}^{2}}{2}
$$

With the sum of squares of ${ }^{x}$ and ${ }^{y}$, the simplification of Eq. (1) can be written as:

$$
\begin{aligned}
& x^{2}+y^{2}=r_{b}\left(1+\varphi^{2}\right) \\
& \text { Set } \rho^{2}=x^{2}+y^{2} . \text { Eq. (5) changes to: } \\
& \varphi=\sqrt{\frac{\rho^{2}}{r_{b}^{2}}-1}
\end{aligned}
$$

Set $\rho=r_{f}=1462.5 \mathrm{~mm}$, then $\varphi_{1}=0.33$. Set $\rho=r_{a}=1501.2 \mathrm{~mm}$, then $\varphi_{2}=0.41$

After introducting $\varphi_{1}=0.33$ and $\varphi_{2}=0.41$ into Eq. (4), the length of the involute is: $L=41.35 \mathrm{~mm}$

(2) Determine the contact area and the surface load

Meshing gears in the process of contact area is a small rectangular surface. The area can be given by:

$S=b t$

where the tooth width, $b=120 \mathrm{~mm}$, and $\mathrm{t}$ is the two gears contact width.

According to Hertz formula:

$$
t=1.128 \times \sqrt{P_{n} \frac{R_{1}}{R_{2}}\left(\frac{1-v_{1}^{2}}{E_{1}}+\frac{1-v_{2}^{2}}{E_{2}}\right)}
$$

where the pitch circle radius of two meshing gears is $R_{1}$ and $R_{2}$, respectively; $P_{n}$ is the load per unit length through the contact width; the Poisson coefficient of the two gears is $v_{1}$ and $v_{2}$, respectively; the elastic modulus of the two gears is $E_{1}$ and $E_{2}$, respectively.

According to material properties, we set $v_{1}=v_{2}=0.3$ and $E_{1}=E_{2}=210 \mathrm{GPa}$ 
The maximum rotation torque for the outer ring gear is $88 \mathrm{kNm}$. When the pressure angle is $20^{\circ}$, the normal force can be calculated as $F_{\mathrm{b} n c}=122.27 \mathrm{kN}$. The load per unit length through the contact width is determined by the normal force and the tooth width.

$$
P_{n}=\frac{F_{\mathrm{b} n c}}{b}=1018.92 \mathrm{~N} / \mathrm{mm}
$$

Then, introduce $P_{n}, R_{1}, R_{2}, v$ and $E$ into Eq. (8), and obtain the value of $\mathrm{t}$ :

$$
t=1.14 \mathrm{~mm}
$$

Thus, the contact area can be calculated as: $S=b \times t=136.8 \mathrm{~mm}^{2}$

The surface loads is:

$$
\frac{F_{\mathrm{b} n c}}{\mathrm{~S}}=\frac{122.27 \mathrm{KN}}{136.8 \mathrm{~mm}^{2}}=893.84 \mathrm{MPa}
$$

(3) Determine the load position

With the overall length of the involute and the contact width, the involute length is divideed into ${ }^{x_{t}}$ segments averagly. According to $\mathrm{xt}=\mathrm{L} / \mathrm{t}=36.2$, the rounding value of

$x_{t}$ is chosen as 36 . Then, the loading area (small rectangle) can be determined by the value of $x / 36$.

4) Set constraints

The yaw bearing is set on the top of the tower and connected with the nacelle and the tower by bolts, as shown in Fig. 1. So, the lower surface of the bearing outer ring is fixed by all directions in FEA. Since only part of the model is analyzed, symmetric constraints are imposed on the profiles.

5) Post-process and analyze

From the calculation results as shown in Fig. 7 and 8, we can find that the maximum bending stress occurs at the tooth fillet under the limit load. The maximum bending stress is 177.512MPa, which is within the allowable stress range, and the maximum deformation value is $0.84 \times 10-10 \mathrm{~m}$. The results show that the main failure form of the yaw bearing is the bending fatigue fracture of the outer ring gear tooth root. Besides, the load on the outter ring gear leads to the surface and total deformation, which affects the load distribution among gear teeth and dynamic performance.

According to the traditional calculation fomulae, the stress is $220.91 \mathrm{MPa}$, which is larger than the result of ANSYS. The reason is that the selection of each factor in the traditional algorithm tends to be large enough to ensure the safety. This fact also verifies that the results of ANSYS are more close to the practice of the outer ring gear in the yaw bearing and provide more accurate indication of the force on the gear.

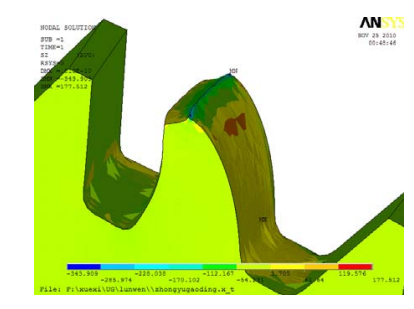

Figure 7. The contour of contact force

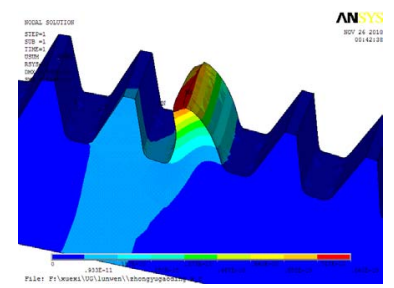

Figure 8 . The contour of contact stress

\section{CONCLUSIONS}

This paper provides the following conclusions:

(1) Parametric modeling of yaw bearings was introduced in this research. It was a very convenient and effective way to use the model parameters for the precise control of profiles. Moreover, we only needed to modify the "expression" dialog box to change parameters, without reediting the formulas. The partial cutting away tool of UG was also applied to easily observe the internal structure, which can ensure the assembly accurately.

(2) A new method was presented here to determine the loading position on the outer ring gear in the process of FEA. It can be found that the loading position determined by this method is more accurate and close to the practice. Therefore, the new method can be used to explain the failure reasons of the gear meshing process well.

(3) Through the statistic analysis in the extrem loading condition, we can obtain the stress and strain in the main parts of the yaw bearing, which provided a new and practical idea for the design and analysis of products.

\section{REFERENCES}

[1] Tony Burton, Wu xin. Wind Energy: Handbook. Science Press [M]. 2007, PP:84-105.

[2] Du Linli, Shi Yong, Guo Xuwei. Proficient in UG NX 4.0. Electronic Industry Press [M], 2006:PP 20-178.

[3] Wen Lige, Hou Hongsheng. Parameterized Design of Cylindrical Involute Helical Gear Using UG. Modern Manufacturing Engineering [J], 2009, 1, PP. 33-35.

[4] IEC 61400-1, Wind turbines Part 1:Design requirements. International Electrotechnical Commission [S], 2005, PP. 47-49.

[5] Zhang Yingqian, Zhang Hongcai. ANSYS Structural Analysis from Novice. Prosts \&Telecom Press [M], 2010, PP. 30-207. 